\title{
Classification and Review of Hand Rehabilitation Devices
}

\author{
Felix ARNAUT \\ Transilvania University of Brasov, Romania, felix.arnaut@unitbv.ro \\ Tudor DEACONESCU \\ Transilvania University of Brasov, Romania, tdeacon@unitbv.ro
}

\begin{abstract}
The aim of this study is to provide a general insight into the causes and mechanisms that lead to hemiparesis while also providing insight into how the condition can be ameliorated or even treated. For the scope of this study, the only treatment methods considered were the ones using robotic rehabilitation devices as opposed to classical methods, such as kinesiotherapy and, where available, provide a comparison between the two. To form a clearer picture of the different types of rehabilitation devices, a classification method based on the device`s interaction with the patient, the type of actuator and it's stiffness is introduced and different examples are provided along with available specifications. The study further compiles data on the development of soft robotics rehabilitation devices and emphasises the effectiveness and adaptiveness of said devices and highlights the importance of additional research in this field.
\end{abstract}

\section{Keywords}

rehabilitation equipment, hemiparesis, soft robotics

\section{Introduction}

Hemiparesis is an affliction whose symptoms include total, or partial loss of motion on one side of the body. The most often cause of hemiparesis is a stroke that affects the brain hemisphere opposite to the impaired side of the body [1]. By obtaining MRI (magnetic resonance imaging) scans of elderly, hemiparetic people $(71 \pm 6$ years) who recently suffered a stroke, researchers found that $92.8 \%$ of the sample group had previously experienced a different stroke and that $85 \%$ of those already had slight motor deficiencies. This indicated that the brain has the ability to compensate for the damaged hemispheres by assigning the tasks to different areas [2].

Besides compensating for the damaged tissue, the brain is also able to mend the area affected by the stroke. This process is made possible if the patient undergoes a routine of recovery therapy and exercise. Using MRI scans, the area affected by an acute cortical stroke can be made visible, before and after therapy, as seen in Fig. 1 [3, 4].
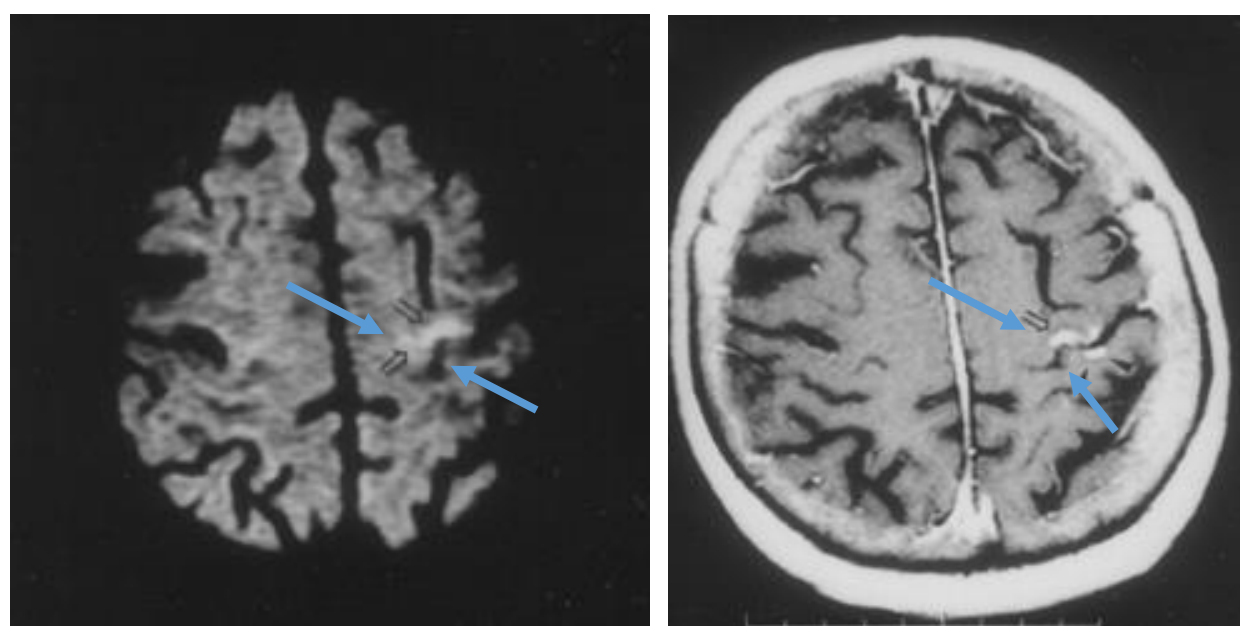

Fig. 1. MRI scans of a patient affected by stroke.

Arrows indicate the affected area before (left) and after (right) therapy 
Traditional rehabilitation methods such as kinesitherapy, mirror therapy, occupational therapy or constraint therapy have evolved continuously since the 1990`s, especially with the development of rehabilitation devices. These devices were initially aimed at assisting the therapist's tasks (such as heavy lifting, strenuous activities), hence as auxiliary equipment. Nowadays, robotic rehabilitation is a self-standing multidisciplinary field $[5,6]$.

\section{Rehabilitation Equipment}

Subsequently to conducting a systematic literature analysis, it was decided to categorize the presented findings by mode of application (exoskeleton or final end), actuator type (electrical or pneumatic) and stiffness of the actuator components (hard robotics or soft robotics).

\subsection{Exoskeleton type rehabilitation devices}

As the name suggests, this kind of device can be worn by the user. Thus, it has to be customizable, or specifically designed to fit each individual user. Such a device is the HandExos [7]. The HandExos works by attaching a polymeric clamp to each phalanx, as seen in Fig. 2. In turn, a cable travels through each clamp to a battery powered DC (direct current) electric motor. Hence a pulley system is created. When the motor increases/weakens cable tension, the finger extends/ flexes [7].
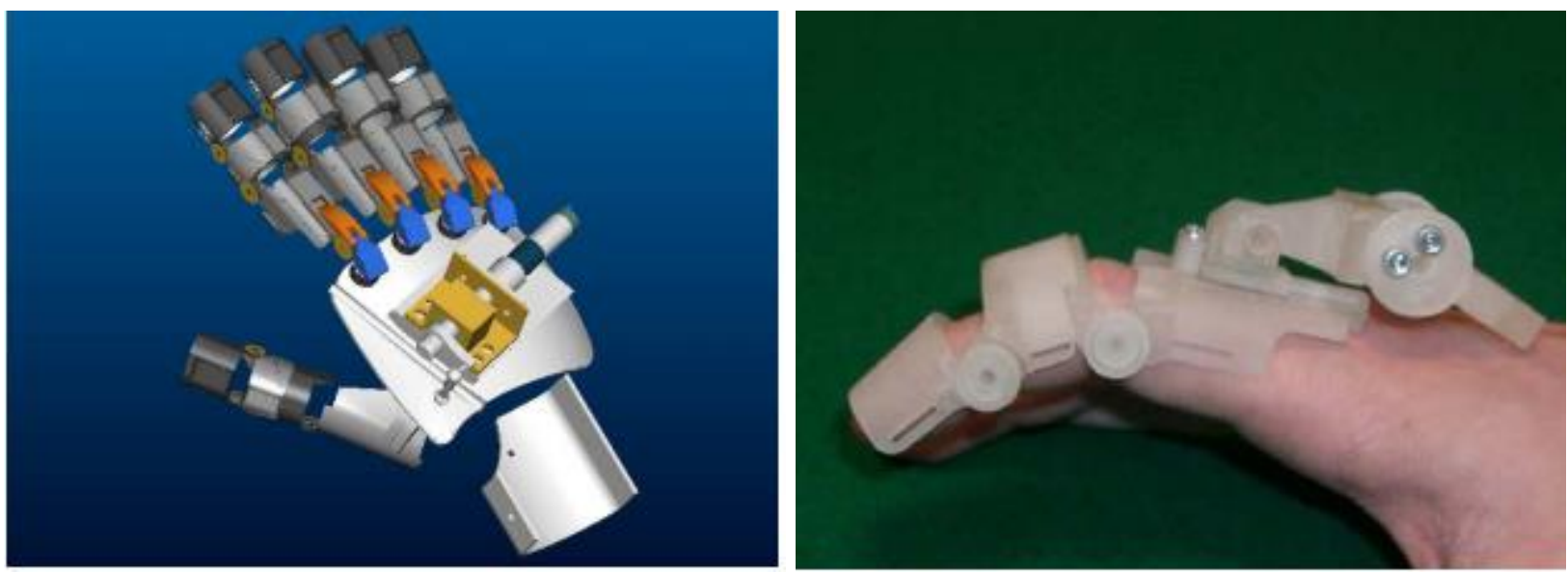

Fig. 2. Schematic (left) and prototype (right) of the HandExos rehabilitation device

This concept was taken even further by researchers from the University of Pittsburgh, who integrated EMG (electromyography) sensors to the previously presented variant. Hence, the patient can control de device by using the electrical signal of their nervous system. This is possible because the instant the patient wants to grab something, the brain sends corresponding signals to the muscles. Since the muscles cannot perform the action, the signal is picked up by the EMG sensors and used to initiate the exoskeleton. As seen in Fig. 3 the patient is able to grab the roll of tape [8].

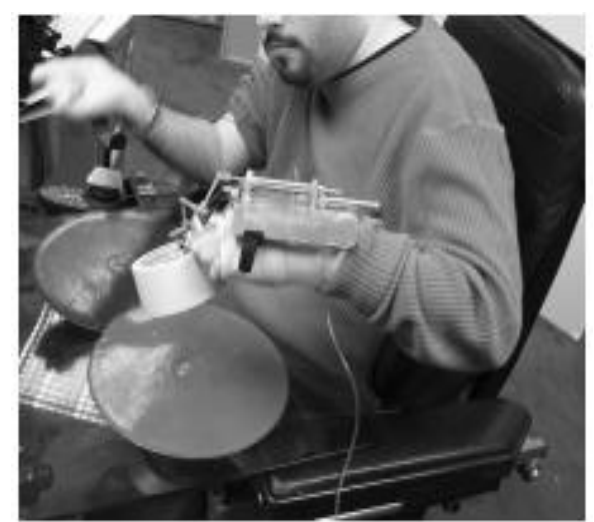

Fig. 3. EMG Exoskeletal device in action 
The main advantage of exoskeletal rehabilitation equipment is that it can be also used in day-to-day activities of the patient, not just during therapy sessions. Thus, the device enables the patients to grab and lift relatively light household objects such as a glass of water. The disadvantage consists in the usage of the device limited by its battery life.

\subsection{Final end type rehabilitation devices (FERDs)}

In the case of FERDs, the patient is strapped on only throughout the therapy session. This kind of devices are cumbersome, bulky and mass produced for use in clinics. Although they are available on the market, the high price, the large size and the requirement of trained personnel makes FERDs unfit for home use. An example of a state of the art FERD is Amadeo, a device built by Tyromotion, an Austrian company, shown in Fig. 4 [9].

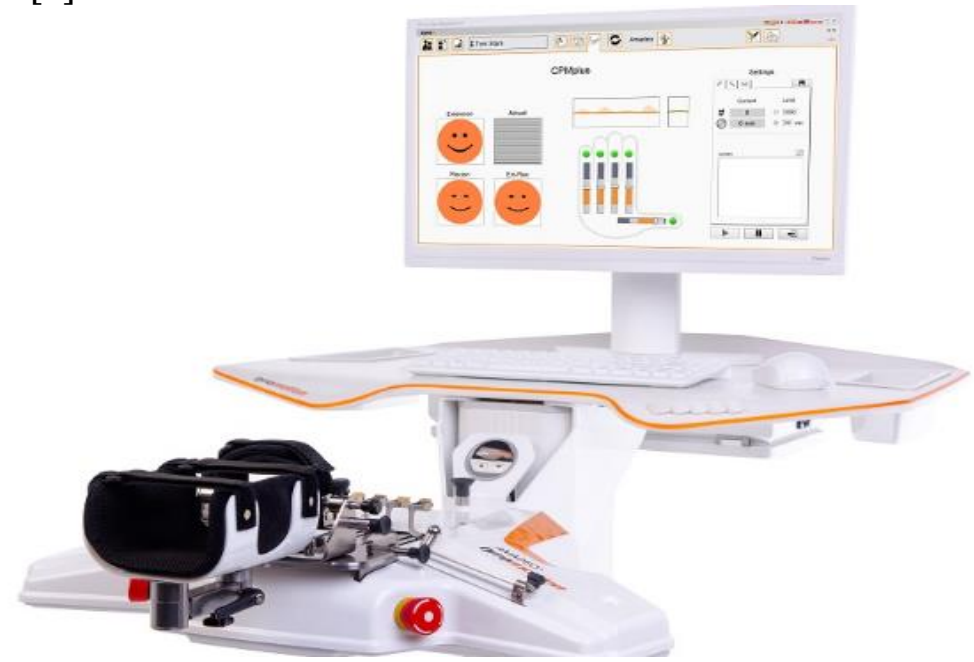

Fig. 4. Amadeo FERD

The device uses straps to hold the patient's forearm in place, while the fingers are placed in specially designed mounts. Each finger can be exercised independently by the sliding of each mount on a rail, parallel to the length of the hand. The hand mount can be seen in Fig. 5, and although it only comes in one size, according to the manufacturer it can fit both adults and children. Another advantage is that Amadeo can also be used as a research and diagnostics tool. Sensors are in place that can determine the force, spasticity, range of movement and muscle strength for each individual finger and relay it to the operator in a user-friendly interface. Moreover, EMC sensors are incorporated that can be used to determine the strength of the nerves signal in the affected muscles. Another advantage of using EMC sensors is that the muscles can be stimulated to perform active rehabilitation exercises, which would otherwise be impossible [9].

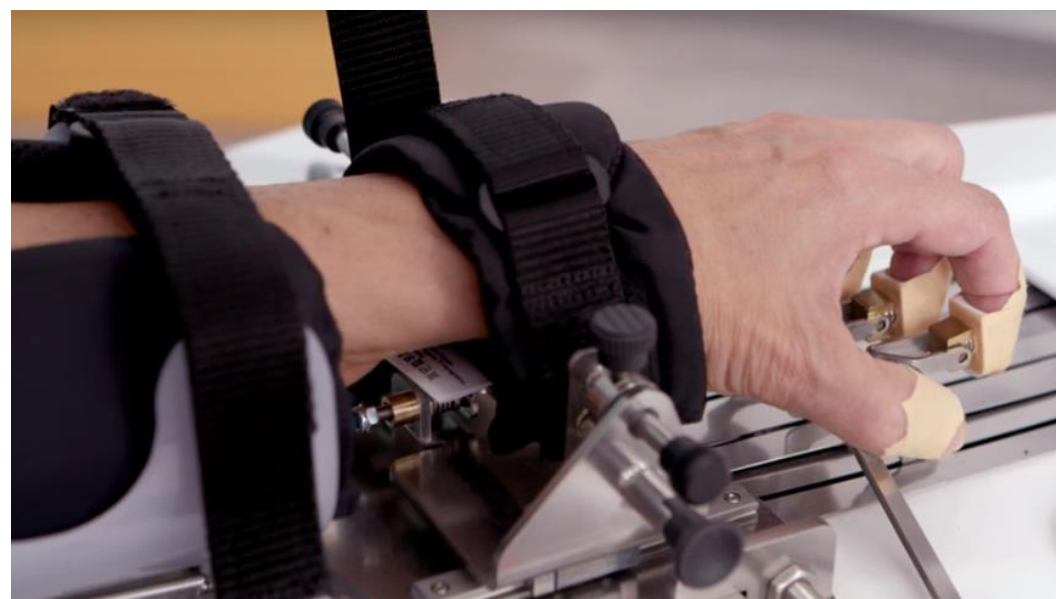

Fig. 5. Patient hand strapped to Amadeo 


\subsection{Electrically actuated rehabilitation devices}

Electrically actuated devices use an electric motor to initialize the action. In the case of applications where small size, mobility and weight are essential criteria, DC motors are the best choice. The main advantage of using electric actuators is their long service life and instant delivery of torque. Additionally, a microcontroller is needed to supply the control signal. Hence, it is possible to connect different sensors to the microcontroller and program a routine based on the data received from said sensors [10].

A device that incorporates all the above advantages is the Gloreha. It uses electrically actuated tendons to move the fingers. These tendons are attached to a glove worn by the patient as seen in Fig. 6 [11].

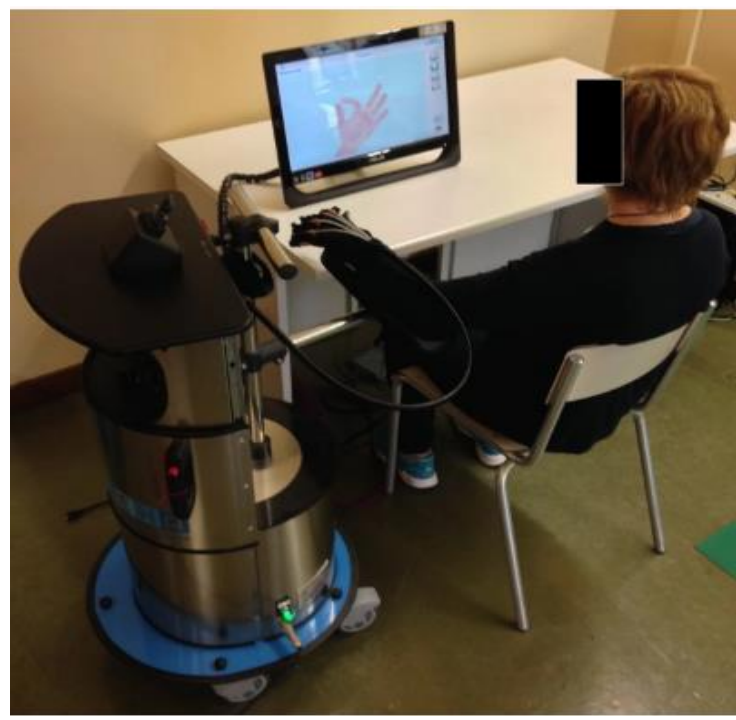

Fig. 6. Gloreha rehabilitation device

This device is available on the market, and has been proven to be highly efficient, having shown to reduce hemiparesis associated spasticity by $69 \%$ [11]. Also, it has been shown to be 3.05 times more efficient at reducing pain, compared to a routine consisting of physiotherapy combined with occupational therapy [12].

The disadvantage of Gloreha is its high price and bulkiness that render it unsuitable for domestic use. The price can reach $30,000 €$ and taking into account the length of a therapy session, during the working hours of a clinic, the device can only be used for an estimate of 67 times a year.

\subsection{Pneumatically actuated rehabilitation devices}

The most common type of pneumatic actuator used for rehabilitation devices is the McKibben. It consists of an elastic membrane wrapped in a mesh of fibres that only allow the membrane to deform longitudinally when the system is pressurized. Researchers propose a number ways to dispose the fibres based on the desired effect, as seen in Fig. $7[13,14]$.
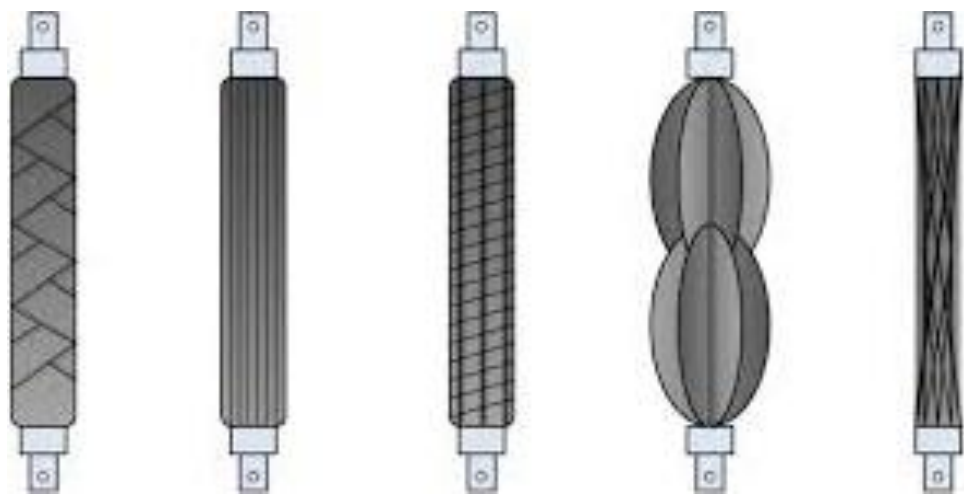

Fig. 7. McKibben muscles obtained using different fibre orientation 
Pneumatic actuators are used for their high power to weight ratio, low cost and operating safety. Moreover, they are easy to install. The challenge, however, is that any pneumatic system requires a compressor to build up pressure. A Japanese team of researchers proposes an innovative solution in the form of an ElectroHydroDynamic micropump in combination with a thin artificial muscle, as seen in Fig. 8. They obtained a system of small dimensions, which weighs $2 \mathrm{~g}$. Despite its size, it can exert a force of $0.84 \mathrm{~N}$ and extend $4 \mathrm{~mm}[15]$.

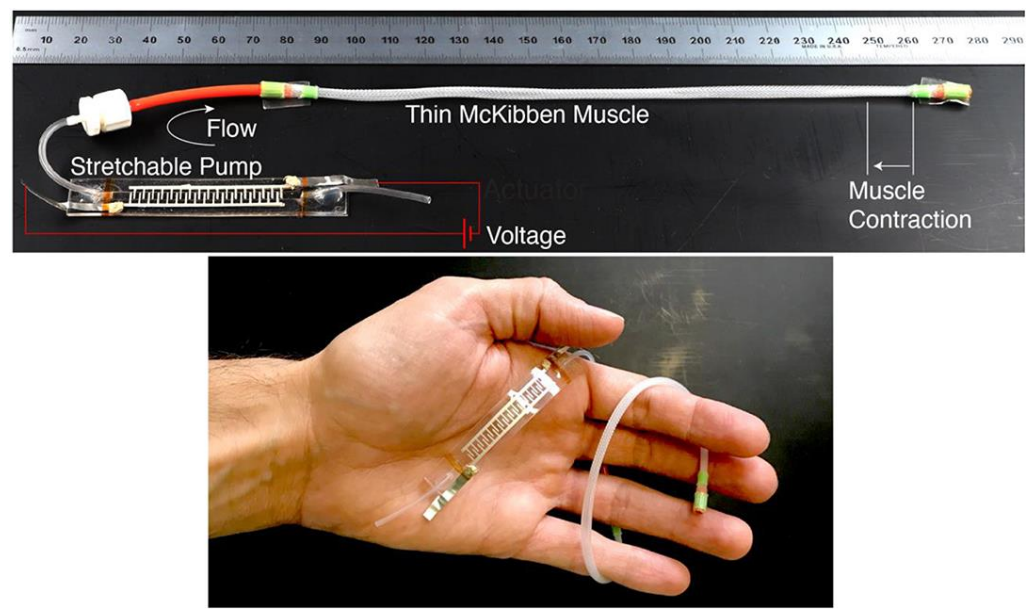

Fig. 8. McKibben muscle actuated by ElectroHydroPumb

\subsection{Soft robotics rehabilitation devices}

Unlike the previously presented rehabilitation devices, which are built from rigid materials, soft robotics aims at using only non-rigid materials mimicking biological systems most of that consist of soft, elastic tissue (with the exception of bone). Hence, there is a general interest in the development of rehabilitation devices that use soft deformable materials $[16,17]$.

For a material to be considered soft, it needs to have an elastic modulus of around $1 \mathrm{GPa}$, which is similar to biological tissue. Most materials used are silicone, rubber and elastic polymers [18].

A simple, yet very interesting soft robotics rehabilitation device is the magneto-rheological MR Glove shown in Fig. 9. It is a specially designed exoskeletal device which enables patients to attend MRI scans while wearing it. The glove consists of a 3D printed pneumatic actuator encased in a textile pocket that only permits the actuator to deform according to the natural motion of the patient's finger. The glove can exert a maximum force of $40 \mathrm{~N}$, enabling the patient to lift a weight of almost $2 \mathrm{~kg}$ [19].

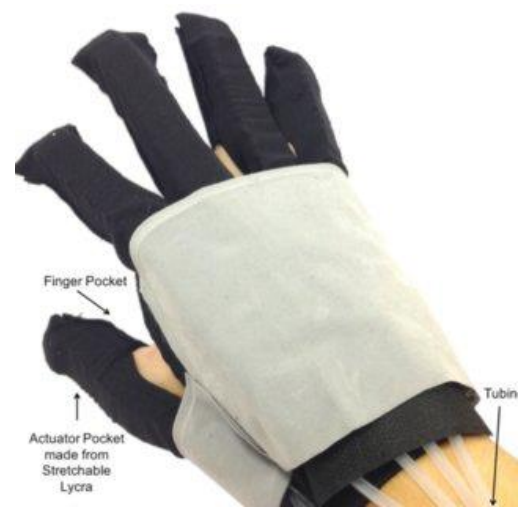

Fig. 9. MR Glove

More recently another team of researchers has put forward an even simpler to build device that includes already existing McKibben muscles attached to a construction glove as seen in Fig. 10. The McKibben actuators are arranged in a pattern similar to that of the human muscles, hence reproducing the natural movements. 


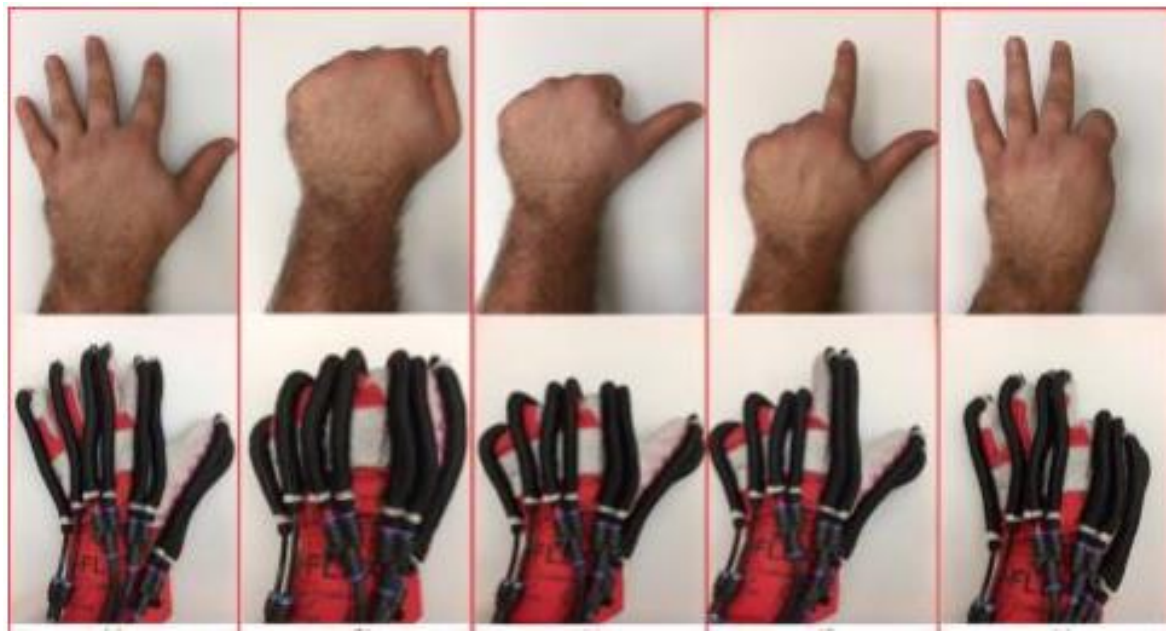

Fig. 10. Soft robotic rehabilitation device

Another characteristic of this device is that due to its EMG module the patient is able to use the glove as desired, not just according to a pre-programmed routine. Also, patient nerve activity in the affected hand can be measured, as in the experiment shown in Fig. 11.

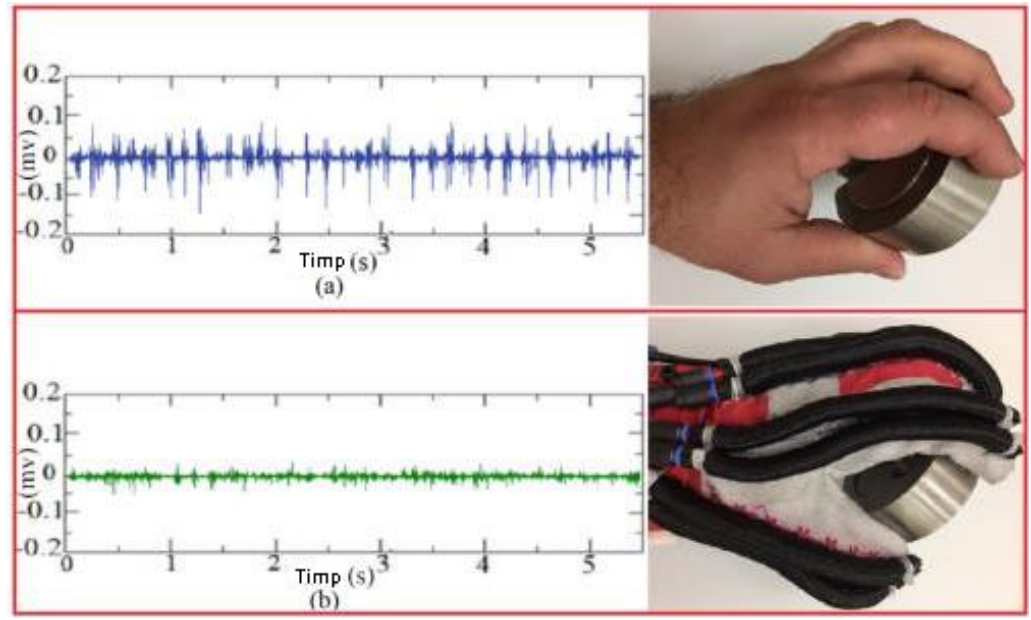

Fig. 11 EMG signal strength in a healthy(top) and hemiparetic patient during a gripping experiment

The task comprised by the experiment was lifting a $1 \mathrm{~kg}$ weight. As expected, the EMG signal of the hemiparetic patient is weaker than that of the healthy one, hence the utility of and need for EMG sensors.

The weight of the glove itself is of $0.19 \mathrm{~kg}$, while the total mass of the system is $4 \mathrm{~kg}$. This makes it usable in patient day-to-day life [20].

\section{Conclusion}

The main aim of this report is to present the mechanisms that lead to hemiparesis and how the condition can be treated. Research has shown that the condition is treatable and that rehabilitation devices can play a vital role. The development of rehabilitation devices has seen numerous breakthroughs in point of both care and medical devices.

Research has also shown that even though classic, hard robotics can be used to develop effective rehabilitation devices, there is an application for the use of soft robotics in the field that offers a whole new range of possibilities.

Additional research in the development of rehabilitation devices and how knowledge from different fields can be integrated is required in order to achieve a better point of care device that patient can use in daily life. Moreover, the development of soft robotics rehabilitation devices should be pursued as they may offer a better alternative to the classic ones. 


\section{References}

1. American Stroke Asociation (2020): Hemiparesis. https://www.stroke.org/en/about-stroke/effects-ofstroke/physical-effects-of-stroke/physical-impact/hemiparesis

2. Inatomi Y., Nakajima M., Yonehara T., Ando Y. (2017): Ipsilateral hemiparesis in ischemic stroke patients. Acta Neurologica Scandinavica, eISSN 1600-0404, Vol. 136, no. 1, pp. 31-40, DOI: 10.1111/ane.12690

3. Back T., Mrowka M. (2001): Infarction of the "hand knob" area. Neurology, eISSN 1526-632X, Vol. 57, no. 6, p. 1143, https://doi.org/10.1212/WNL.57.6.1143

4. Boukadida A., Piotte F., Dehail P., Nadeau S. (2015): Determinants of sit-to-stand tasks in individuals with hemiparesis post stroke: A review. Annals of physical and rehabilitation medicine, ISSN 1877-0657, eISSN 1877-0665, Vol. 58, no. 3, pp. 167-172, DOI: 10.1016/j.rehab.2015.04.007

5. Yue Z., Zhang X., Wang J. (2017): Hand Rehabilitation Robotics on Poststroke Motor Recovery. Behavioural Neurology, eISSN 1875-8584, Special Issue: Neural Engineering for Rehabilitation, Vol. 2017, pp. 2-20, Article ID 3908135, https://doi.org/10.1155/2017/3908135

6. Dipietro L., Ferraro M. et al. (2005): Customized interactive robotic treatment for stroke: EMG-triggered therapy. IEEE Transactions on Neural Systems and Rehabilitation Engineering, ISSN 1534-4320, eISSN 1558-0210, Vol. 13, is. 3, pp. 325-334, doi: 10.1109/TNSRE.2005.850423

7. Chiri A., Giovacchini F., et al. (2008): Design of a hand exoskeleton (HANDEXOS) for the rehabilitation of the hand. Gerontechnology, https://journal.gerontechnology.org/archives/2008papers/168.pdf

8. Lucas L., DiCicco M., Matsuoka Y. (2004): An EMG-Controlled Hand Exoskeleton for Natural Pinching. Journal of Robotics and Mechatronics, eISSN 1883-8049, Vol.16, no. 5, pp. 482-488, DOI:10.20965/jrm.2004.p0482

9. *** (2020): Amadeo, The Hand Therapy World Champion. https://tyromotion.com/en/products/amadeo/

10. Zhang P. (2010): Advanced Industrial Control Technology. Elsevier, ISBN 978-1-4377-7807-6,

11. Bissolotti L., Villafañe J.H., et al. (2016): Changes in skeletal muscle perfusion and spasticity in patients with poststroke hemiparesis treated by robotic assistance (Gloreha) of the hand. Journal of physical therapy science, ISSN 0915-5287, eISSN 2187-5626, Vol. 28, no. 3, pp. 769-773, https://doi.org/10.1589/ipts.28.769

12. Villafañe J.H., Taveggia G., et al. (2018): Efficacy of Short-Term Robot-Assisted Rehabilitation in Patients with Hand Paralysis after Stroke: A Randomized Clinical Trial. Hand (N Y), ISSN 1558-9447eISSN 1558-9455, Vol. 13, no. 1, pp. 95-102, https://doi.org/10.1177/1558944717692096

13. Liu W., Rahn C.R. (2004): Fiber-Reinforced Membrane Models of McKibben Actuators. Journal of Applied Mechanics, eISSN 1528-9036, Vol. 70, is. 6, pp. 853-859, https://doi.org/10.1115/1.1630812

14. Andrikopoulos G., Nikolakopoulos G., Manesis S. (2011): A Survey on applications of Pneumatic Artificial Muscles. 19th Mediterranean Conference on Control and Automation (MED), ISBN 978-1-4577-0123-8, pp. 1439-1446

15. Cacucciolo V., Nabae H., Suzumori K., Shea H. (2020): Electrically-Driven Soft Fluidic Actuators Combining Stretchable Pumps with Thin McKibben Muscles. Frontiers in Robotics and AI, eISSN 2296-9144, Vol. 6, article 146, https://doi.org/10.3389/frobt.2019.00146

16. Iida F., Laschi C. (2011): Soft Robotics: Challenges and Perspectives. Procedia Computer Science, eISSN 18770509, Vol. 7, pp. 99-102, https://doi.org/10.1016/i.procs.2011.12.030

17. Laschi C., Cianchetti M. (2014): Soft Robotics: New Perspectives for Robot Bodyware and Control. Frontiers in Bioengineering and Biotechnology, eISSN 2296-4185, Vol. 2, article 3, DOI: 10.3389/fbioe.2014.00003,

18. Hughes J., Culha U., et al. (2016): Soft Manipulators and Grippers: A Review. Frontiers in Robotics and AI, eISSN 2296-9144, Vol. 3, article 69, https://doi.org/10.3389/frobt.2016.00069

19. Yap H.K., Kamaldin N., et al. (2017): A Magnetic Resonance Compatible Soft Wearable Robotic Glove for Hand Rehabilitation and Brain Imaging. IEEE Transactions on Neural Systems and Rehabilitation Engineering, eISSN 1558-0210, Vol. 25, is. 6, pp. 782-793, DOI: 10.1109/TNSRE.2016.2602941

20. Al-Fahaam H.S.H. (2019): Wearable exoskeleton systems based-on pneumatic soft actuators and controlled by parallel processing. $\mathrm{PhD}$ thesis, University of Salford, http://usir.salford.ac.uk/id/eprint/51875 